\title{
Sono e adolescência: quantas horas os adolescentes precisam dormir?
}

\author{
Sleep and adolescence: how many hours sleep teenagers need? \\ Érico Felden Pereira', Diego Grasel Barbosa', Rubian Diego Andrade', Gaia Salvador Claumann', \\ Andreia Pelegrini', Fernando Mazzilli Louzada²
}

\section{RESUMO}

Objetivo: Determinar a especificidade e a sensibilidade de uma medida para apontar o meIhor ponto de corte para a duração de sono como preditor da sonolência diurna excessiva em adolescentes. Métodos: Participaram do estudo 1.359 adolescentes, com idades de 14 a 21 anos, de duas cidades do sul do Brasil, que responderam a questionário de hábitos de sono e sonolência diurna. Utilizou-se a Receiver Operating Characteristic para estimar a capacidade preditiva da duração de sono para a sonolência diurna excessiva. Resultados: A média de duração do sono para os adolescentes com sonolência diurna excessiva foi de 7,9 horas e para aqueles sem sonolência diurna excessiva foi de 8,33 horas $(p<0,001)$. A prevalência de sonolência diurna excessiva foi de $35,7 \%$. Foi observada correlação significativa e negativa entre a duração do sono e as idades analisadas $(p<0,001)$. A análise de Receiver Operating

\section{Palavras-chave}

Sono, distúrbios do sono por sonolência excessiva, adolescentes.

\section{Keywords}

Sleep, disorders of excessive somnolence, adolescents.
Characteristic indicou duração mínima de 8,33 horas como proteção para a sonolência diurna excessiva. Conclusão: Foi observada alta prevalência de sonolência diurna excessiva e propõe-se como possível duração de sono um mínimo de 8,33 horas nos dias com aula para que os adolescentes evitem esse desfecho.

\section{ABSTRACT}

Objective: Determine specificity and sensibility, to point the best cut off for the sleep duration as predictor of excessive daytime somnolence in adolescents. Methods: One thousand three hundred fifty-nine adolescents aged 14 to 21 years of two cities from South Brazil answered a questionnaire about sleep habits and daytime somnolence. To estimate the predictive capacity of sleep duration for the excessive daytime somnolence was used the Receiver Operating Characteristic. Results: The sleep duration mean for adolescents with excessive daytime somnolence was 7.9 hours and for those without excessive daytime somnolence was 8.33 hours $(p<0,001)$. The prevalence of excessive daytime somnolence was $35.7 \%$. Significant and negative correlation between the sleep duration and the ages analyzed was observed $(p<0,001)$. The Receiver Operating Characteristic analysis indicated a minimum duration of 8.33 hours as protection for excessive daytime somnolence. Conclusion: High prevalence of excessive daytime somnolence was observed and is proposed as a possible sleep duration a minimum of 8.33 hours of sleep in the days with classes for adolescents to avoid this outcome.

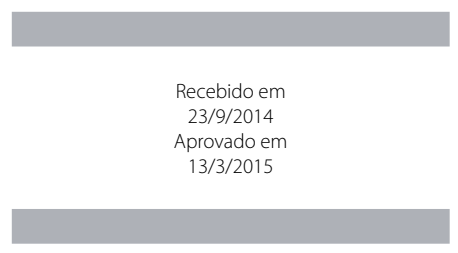

DOI: 10.1590/0047-2085000000055
1 Universidade do Estado de Santa Catarina (UDESC).

2 Universidade Federal do Paraná (UFPR).

Endereço para correspondência: Érico Felden Pereira

Universidade do Estado de Santa Catarina (UDESC), Centro de Ciências da Saúde e do Esporte (CEFID)

Rua Pascoal Simone, 358, Coqueiros

88080-350 - Florianópolis, SC, Brasil

E-mail: ericofelden@gmail.com 


\section{INTRODUÇÃO}

O sono é um dos principais processos fisiológicos para a vida. A sua expressão, alternada com a vigília, é circadiana e sofre influência de fatores endógenos, sociais e ambientais'. Os adolescentes estão sujeitos a alterações de seu padrão de sono por causa da maturação e dos horários e demandas estabelecidos, especialmente, pelas instituições de ensino². Em termos biológicos, os adolescentes podem apresentar atraso da fase de sono, caracterizado por horários mais tardios de dormir e acordar ${ }^{3}$, além de modificações na duração de alguns estágios de sono, como o sono de ondas lentas4.

Pesquisas epidemiológicas vêm mostrando crescentes prevalências de baixa duração do sono nos adolescentes e associações desse débito com inúmeros desfechos na saúde, incluindo problemas metabólicos e $\operatorname{cognitivos}^{5-8}$. A sonolência diurna excessiva, caracterizada como sensação aumentada da necessidade de sono e diminuição do estado de alerta ${ }^{9}$, é um dos principais efeitos da duração reduzida de sono ou de sono de baixa qualidade. Trata-se de um problema de saúde pública tendo em vista as altas prevalências identificadas na população, especialmente de adolescentes, podendo chegar a $68 \%{ }^{10}$

Para Wolfson et al."11, a privação crônica de sono em adolescentes e jovens é um problema global e multifatorial. Ele apresenta uma possível recomendação de 9 horas de sono para adolescentes e discute que, geralmente, essa quantidade de horas não é alcançada. Já na fase adulta a recomendação de sono, que normalmente gira em torno de 8 horas, também não está sendo alcançada por boa parte da população.

Mesmo sabendo dos efeitos da privação de sono para o aumento da sonolência diurna excessiva e que, na adolescência, a recomendação de horas de sono deve ser superior quando comparada com a vida adulta, verifica-se carência de estudos que apresentem dados mais precisos com relação à quantidade ideal de sono a ser utilizada em recomendações epidemiológicas. Destaca-se que em termos individuais a duração adequada de sono é singular para cada pessoa, mas, apesar disso, no âmbito da saúde pública, são de suma importância para a saúde dos adolescentes possíveis recomendações mínimas da quantidade de horas de sono, necessárias para melhor aproveitamento e rendimento escolar. Assim, o principal objetivo deste estudo foi determinar a especificidade e a sensibilidade de uma medida para apontar o melhor ponto de corte para a duração de sono como preditor da sonolência diurna excessiva em adolescentes.

\section{MÉTODOS}

Trata-se de uma análise secundária de dados realizada com base em duas pesquisas com adolescentes. A pesquisa 1 (amostra 1) foi realizada com adolescentes do ensino médio do município de Santa Maria (RS); a pesquisa 2 (amostra 2) foi realizada com amostra de universitários ingressantes de uma universidade do sul do Brasil. Ambas as pesquisas foram analisadas e aprovadas pelo Comitê de Ética em Pesquisa com Seres Humanos. As descrições detalhadas das amostras são apresentadas a seguir. Todos os adolescentes concordaram em participar da pesquisa e assinaram o termo de consentimento livre e esclarecido. Para os menores de idade, o termo de consentimentos livre e esclarecido foi assinado pelos pais e/ou responsáveis.

A amostra 1 foi constituída por 1.126 estudantes do ensino médio de escolas públicas, sendo 55,1\% do sexo feminino. Trata-se de uma amostra representativa de adolescentes do ensino médio do município de Santa Maria (RS), tendo como base de cálculo dados da secretaria de educação do município. O tamanho dessa amostra foi calculado utilizando a proposta de Luiz e Magnanini'12, considerando-se um erro amostral de três pontos percentuais, prevalência de 50\% (prevalência desconhecida) para pesquisas originais, efeito de delineamento de 1,5 e percentual de 15\% para perdas. Os adolescentes foram selecionados em escolas das diferentes regiões geográficas do município.

A amostra 2 foi constituída com base no número de alunos ingressantes no ensino superior em uma universidade do sul do Brasil, sendo formada por 233 estudantes universitários ingressantes nos cursos de Licenciatura em Educação Física, Bacharelado em Educação Física, Fisioterapia, Administração Empresarial, Administração Pública, Economia, História e Pedagogia da cidade de Florianópolis (SC), sendo 56,2\% do sexo feminino. Para o cálculo dessa amostra, também foram utilizados os procedimentos sugeridos por Luiz e Magnanini'2, com erro amostral de 4\% e efeito de delineamento de 1,5\%.

Em ambas as amostras, foram considerados como adolescentes para a constituição da amostra final desta pesquisa os jovens com até 21 anos.

\section{Instrumentos utilizados}

O mesmo instrumento de avaliação do sono e da sonolência diurna excessiva foi utilizado nas duas amostras. A duração do sono foi inferida com base no tempo na cama. Assim, para obter a duração de sono dos adolescentes, foi aplicado um questionário de hábitos do sono ${ }^{13}$ considerando os horários de dormir e acordar nos dias com aula.

A sonolência diurna foi avaliada por meio da escala de sonolência de Epworth ${ }^{14}$, na qual são descritas oito situações cotidianas (sentado e lendo; vendo TV; sentado em um lugar público; como passageiro em trem, carro ou ônibus (por 1 hora sem parar); deitando-se à tarde para descansar; sentado e conversando com alguém; sentado calmamente após o almoço, sem álcool; enquanto está parado no trânsito, por alguns minutos, considerando uma escala de zero (nenhuma chance) a três (alta chance) relativa à chance que apresenta 
para cochilar em cada uma das situações. Foram classificados com sonolência diurna excessiva aqueles adolescentes com pontuação de 10 ou mais na escala de Epworth ${ }^{15}$.

\section{Análise de dados}

Para análise descritiva, foram usadas medidas descritivas com médias, desvios-padrão e distribuição de frequências. A diferença das médias da duração do sono entre os grupos com e sem sonolência diurna excessiva foi calculada por meio do teste de Kruskal-Wallis. Foi utilizada a curva Receiver Operating Characteristic $(\mathrm{ROC})$ para estimar a capacidade preditiva da duração do sono, utilizando como referência a sonolência diurna excessiva. A partir dessa análise, foram apresentados os índices: área sob a curva ROC, valores de sensibilidade e especificidade e pontos de corte de duração de sono para cada idade investigada (14 a 21 anos). Além disso, foram calculadas razões de prevalências, com associação das variáveis idade e sonolência diurna excessiva, considerando os pontos de corte indicados na curva ROC por meio da regressão de Poisson. Adotou-se um nível de confiança de 95\% ( $p<0,05)$.

\section{RESULTADOS}

Os dados descritivos da amostra foram apresentados na tabela 1. Identificou-se uma média de duração do sono de 8,2 horas. Além disso, os adolescentes investigados apresentaram média de horário de dormir às 22,7 $(3,9)$ horas e horário de acordar às $7,7(1,7)$ horas. Aproximadamente, um terço da amostra foi classificado com sonolência diurna excessiva (10 ou mais pontos na escala de Epworth), e a média da escala de sonolência foi de 8,1 $(3,9)$ pontos.

Os adolescentes investigados que foram classificados com sonolência diurna excessiva apresentaram média de duração do sono de $7,9(1,6)$ horas; já aqueles sem sonolência diurna excessiva apresentaram duração média do sono de $8,3(1,7)$ horas $(p<0,001)$ (Figura 1$)$.

Tabela 1. Dados descritivos da amostra

\begin{tabular}{lc}
\hline Variáveis & Índices* \\
\hline $\mathbf{n}=1.359$ & \\
\hline Idade, anos & $16,5(1,5)$ \\
Idades, \% & \\
14 anos & 8,0 \\
15 anos & 18,7 \\
16 anos & 25,7 \\
17 anos & 23,2 \\
18 anos & 14,5 \\
19 anos & 5,6 \\
20 anos & 21 anos \\
Duração do sono, h & 2,9 \\
Sonolência diurna excessiva, \% & 1,5 \\
\hline
\end{tabular}

*Valores expressos em média (desvio-padrão) ou frequência absoluta e relativa.

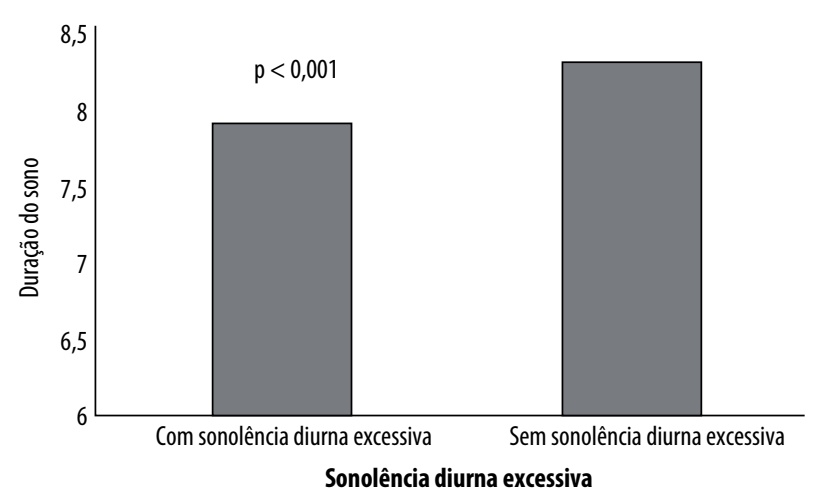

Figura 1. Médias da duração do sono entre adolescentes com e sem sonolência diurna excessiva.

Na figura 2 foi ilustrada a correlação negativa $(r=-0,176$; $p<0,001)$ observada entre a média da duração do sono e a idade dos adolescentes. Nessa análise é possível observar tendência de diminuição progressiva da média da duração do sono ao longo da adolescência. Dessa forma, os adolescentes mais velhos apresentaram duração do sono menor que os mais novos.

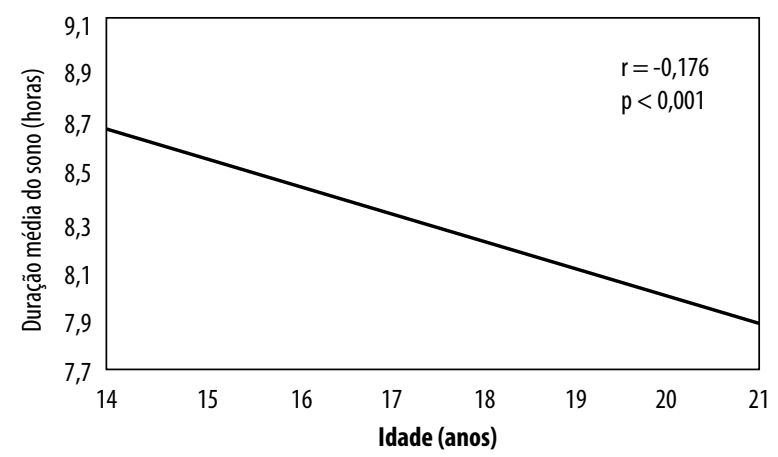

Figura 2. Correlação entre a duração média do sono e a idade.

Na tabela 2 foram apresentados os índices de sensibilidade e especificidade para a proposta de possíveis pontos de corte para a duração de sono de adolescentes, considerando como referência a sonolência diurna excessiva. Primeiramente foi calculada a curva ROC para a amostra total, que identificou necessidade mínima de 8,33 horas de sono dos 14 aos 21 anos. Esse valor possui valor de sensibilidade de $67,8 \%$ e de especificidade de $46,0 \%$, ou seja, o ponto de corte proposto tem maior poder de identificação dos verdadeiros-positivos do que falsos-negativos.

Após, foram calculadas as curvas ROC para cada idade analisada, sendo possível a identificação de pontos de corte nas idades de 16, 17, 19 e 21 anos. Considerando as idades nas quais foi possível identificar o ponto de corte, identificaram-se necessidades de duração do sono maiores que 8 horas na maior parte das idades. A regressão de Poisson na faixa etária de 14 a 21 anos revelou que quem dorme menos 
Tabela 2. Capacidade preditiva da duração do sono em horas considerando a sonolência diurna excessiva como referência

\begin{tabular}{lccccc}
\hline \multirow{2}{*}{ Indicadores } & \multicolumn{4}{c}{ Idade (anos) } \\
\cline { 2 - 6 } & $14-21$ & 16 & 17 & 19 & 21 \\
\hline Área (IC95\%) & 0,574 & 0,571 & 0,598 & 0,661 & 0,761 \\
p-valor & $<0,001$ & 0,035 & 0,003 & 0,0137 & 7,5 \\
Ponto de corte & 8,33 & 8,33 & 8,25 & 0,025 & 8,17 \\
Sensibilidade (IC 95\%) & $67,8(63,2-72,1)$ & $76,7(67,3-84,5)$ & $71,6(62,1-79,8)$ & $63,0(42,4-80,6)$ & $90,9(58,7-98,5)$ \\
Especificidade (IC 95\%) & $46,0(42,5-49,5)$ & $39,1(32,4-46,1)$ & $45,7(38,2-53,4)$ & $72,1(56,3-84,7)$ & $62,5(24,7-91,0)$ \\
RP & 1 & 1 & 1 & 1 & 1 \\
(IC 95\%) & $1,6(1,6-1,7)$ & $1,7(1,5-1,9)$ & $1,6(1,5-1,7)$ & $2,5(2,2-2,9)$ & $4,7(3,2-7,0)$ \\
\hline
\end{tabular}

Para as idades de 14 anos $(p=0,346), 15$ anos $(p=0,265), 18$ anos $(p=0,836)$ e 20 anos $(p=0,075)$ não foi possível estabelecer pontos de corte específicos.

que 8,33 horas por dia tem $60 \%$ mais chances de apresentar sonolência diurna excessiva. Especificamente as idades de 19 e 21 anos foram as que apresentaram maiores razões de prevalência, com índices de 2,5 e 4,7, respectivamente.

\section{DISCUSSÃO}

Na tentativa de minimizar as condições negativas de saúde relacionadas à privação do sono durante a adolescência, entre elas a sonolência diurna excessiva, existe a necessidade da proposta de pontos de corte, como apresentados no corrente estudo. Destaca-se, ainda, que a maior parte dos estudos de cunho epidemiológico sobre o sono na adolescência foi realizada nos Estados Unidos, Europa e países asiáticos e que os dados de sono em populações brasileiras são restritos. A partir dos dados analisados neste estudo, identificou-se que existe prevalência alta de adolescentes com sonolência diurna excessiva e que uma possível recomendação seria de, no mínimo, 8,33 horas de sono nos dias com aula.

A necessidade de sono é individual. No entanto, o estabelecimento de parâmetros gerais de recomendação para adolescentes faz-se necessário para subsidiar recomendações em saúde coletiva. Especialmente na adolescência, a irregularidade na expressão do ciclo vigília/sono ou a privação parcial de sono podem ser percebidas pela diminuição do estado de alerta e concentração no desenvolvimento de atividades acadêmicas ${ }^{16}$. Nesse contexto, estudos apontam tendência de diminuição das horas de sono ao longo da adolescência e elevadas prevalências de adolescentes com baixa duração de sono ${ }^{17-20}$. De acordo com Dollman et al. ${ }^{17}$, o aumento do trabalho para os adolescentes mais velhos e a maior disponibilidade de computadores, televisores e videogames nos quartos contribuíram para exacerbar essa tendência. Apesar disso, a literatura é restrita na indicação precisa de quantas horas os adolescentes precisam dormir.

Assim como a presente pesquisa, diversos estudos buscaram associar a sonolência diurna excessiva com a duração do sono de adolescentes. Estudo ${ }^{18}$ conduzido em 163 crianças e adolescentes alemães, com idades de 6,6 a 17,8 anos, mostrou a duração do sono como preditora da sonolência diurna excessiva. As crianças que apresentaram duração do sono inferior a 8 horas tiveram aumento significativo nos escores do teste de sonolência pupilográfica. Dessa forma, embora a sonolência diurna excessiva seja um fenômeno multifatorial, a baixa duração do sono constitui-se em um de seus principais preditores.

No mesmo sentido, estudo epidemiológico ${ }^{6}$ sobre problemas do sono de 1.939 adolescentes do norte de Taiwan, nas faixas etárias de 12 a 18 anos, revelou média de duração do sono de $7,35(1,23)$ horas, com correlação significativa com a sonolência diurna excessiva nas faixas etárias de 14 a 15 anos $(p=0,001)$ e de 16 a 18 anos $(p=0,003)$.

A prevalência de sonolência diurna excessiva identificada no presente estudo $(35,7 \%)$ foi superior à observada no estudo de Lund et al. ${ }^{7}$, que identificou, em 1.125 estudantes universitários norte-americanos, com idade entre 17 e 24 anos, prevalência de $25 \%$. Já nos estudos de Gibson et al. ${ }^{10} \mathrm{e}$ Chung e Cheung ${ }^{21}$ foi observada prevalência de sonolência diurna excessiva em adolescentes em torno de 40\%. Em todos os casos as prevalências observadas indicam a necessidade de intervenções considerando a saúde do adolescente.

Grande parte dos estudos publicados indica que os adolescentes dormem menos que 9 horas em dias de semana. Loessl et al.22, em pesquisa com 818 escolares do sudoeste da Alemanha, com idades de 12 a 18 anos, revelaram duração de sono média durante a semana de $8,04(0,89)$ horas e correlação entre duração do sono e sonolência diurna excessiva $(p=0,001)$. Considerando a média de sono identificada por Loessl et al..$^{22}$ e o ponto de corte identificado por meio da curva ROC (8,33 horas), poder-se-ia sugerir um aumento de 19,8 minutos de duração de sono para que esses adolescentes tivessem maior proteção para a sonolência diurna excessiva.

No estudo de Liu e Zhou ${ }^{23}$ sobre associações da duração de sono, insônia e problemas comportamentais de 1.359 adolescentes chineses, com idades de 12 a 18 anos, identificou-se que os adolescentes com menos de 7 horas de sono por dia apresentaram maiores prevalências de problemas emocionais. Mesmo que não tenham sido avaliados insônia e proble- 
mas emocionais no presente estudo, sugere-se que 7 horas não sejam a recomendação adequada para essa faixa etária.

No estudo de Carskadon et al. ${ }^{24}$, foi apresentada recomendação de que os adolescentes necessitam em torno de 9 a 10 horas de sono por noite para a manutenção da saúde física e cognitiva. Mas recomendações dessa natureza dependem de condições externas, como em contextos ambientais e diferentes nacionalidades, onde se está inserido ${ }^{25,26}$. O presente estudo é pioneiro em propor pontos de corte de duração de sono para adolescentes no Brasil. As principais limitações dessa análise referem-se à impossibilidade de estabelecer pontos específicos em cada idade analisada. Além disso, outros parâmetros como o grau de alerta devem ser testados, especialmente com medidas diretas como referência para análise de possíveis pontos de corte para a duração de sono com maiores indicadores de sensibilidade e especificidade.

\section{CONCLUSÃO}

De forma geral, com base nos estudos e dados apresentados, pôde-se perceber que a sonolência diurna excessiva é frequente em adolescentes, e a baixa duração do sono é uma das principais causas. No entanto, há carência de estudos que investiguem e estabeleçam pontos de corte entre a duração do sono e a sonolência diurna excessiva, a fim de embasar futuras propostas e/ou intervenções na higiene de sono desse público. Assim, os dados sustentam que os adolescentes necessitam dormir, pelo menos, 8,33 horas nos dias com aula como atitude comportamental para a proteção da sonolência diurna excessiva.

\section{CONTRIBUIÇÕES INDIVIDUAIS}

Todos os autores do presente estudo contribuíram significativamente na concepção e desenho do estudo, na análise e interpretação dos dados, assim como na elaboração do artigo, na revisão crítica do conteúdo intelectual, com aprovação da versão final a ser publicada.

\section{CONFLITOS DE INTERESSE}

Nada a declarar.

\section{REFERÊNCIAS}

1. Foster RG, Wulff K. The rhythm of rest and excess. Nature Rev Neurosci. 2005;6(5):407-14.
2. Pereira EF, Moreno C, Louzada FM. Increased commuting to school time reduces sleep duration in adolescents. Chronobiol Int. 2014;31(1):87-94.

3. Carskadon MA, Acebo C, Jenni OG. Regulation of adolescent sleep: implications for behavior. In: Dahl RE, Spear LP, editors. Adolescent brain development: vulnerabilities and opportunities. New York: Annals of the New York Academy of Sciences; 2004. p. 276-91.

4. Kurth S, Jenni OG, Riedner BA, Tononi G, Carskadon MA, Huber R. Characteristics of sleep slow waves in children and adolescents. Sleep. 2010;33(4):475-80.

5. Liu X, Zhao Z, Jia C, Buysse DJ. Sleep patterns and problems among Chinese adolescents. Pediatrics. 2008;121(6):1165-73.

6. Huang YS, Wang CH, Guilleminault C. An epidemiologic study of sleep problems among adolescents in North Taiwan. Sleep Med. 2010;11(10):1035-42.

7. Lund $H G$, Reider BD, Whiting AB, Prichard JR. Sleep patterns and predictors of disturbed sleep in a large population of college students. J Adolesc Health. 2010;46(2):124-32.

8. Mak KK, Lee SL, Ho SY, Lo WS, Lam TH. Sleep and academic performance in Hong Kong adolescents. J School Health. 2012;82(11):522-7.

9. Hoban TF, Chervin RD. Assessment of sleepiness in children. Semin Pediatr Neurol. 2001;8(4):216-28.

10. Gibson ES, Powles ACP, Thabane L, O'Brien S, Molnar DS, Trajanovic N, et al. "Sleepiness" is serious in adolescence: two surveys of 3235 Canadian students. BMC Public Health. 2006;6:116.

11. Wolfson AR, Spaulding NL, Dandrow C, Baroni EM. Middle school start times: the importance of a good night's sleep for young adolescents. Behav Sleep Med. 2007;5(3):194-209.

12. Luiz RR, Magnanini MM. A lógica da determinação do tamanho da amostra em investigações epidemiológicas. Cad Saúde Colet (Rio J). 2000;8(2):9-28.

13. Louzada F, Menna-Barret L. Sleep-wake cycle in rural populations. Biol Rhythm Res. 2004;35(1-2):153-7.

14. Johns MW. A new method for measuring daytime sleepiness - the Epworth Sleepiness Scale. Sleep. 1991;14(6):540-5.

15. Johns MW. Sensitivity and specificity of the Multiple Sleep Latency Test (MSLT), the maintenance of wakefulness test and the Epworth sleepiness scale: failure of the MSLT as a gold standard. J Sleep Res. 2000;9(1):5-11.

16. Lima PF, Medeiros ALD, Araujo JF. Sleep-wake pattern of medical students: early versus late class starting time. Braz J Med Biol Res. 2002;35(11):1373-7.

17. Dollman I, Ridley K, Olds T, Lowe E. Trends in the duration of school-day sleep among 10-to 15-year-old South Australians between 1985 and 2004. Acta Paediatr. 2007;96(7):1011-4.

18. Randler C, Bilger S, Francisco Diaz-Morales J. Associations among sleep, chronotype, parental monitoring, and pubertal development among German adolescents. J Psychol. 2009;143(5):509-20.

19. Colrain IM, Baker FC. Changes in sleep as a function of adolescent development. Neuropsychol Rev. 2011;21(1):5-21.

20. Matricciani L, Olds T, Petkov J. In search of lost sleep: secular trends in the sleep time of school-aged children and adolescents. Sleep Med Rev. 2012;16(3):203-11.

21. Chung KF, Cheung MM. Sleep-wake patterns and sleep disturbance among Hong Kong Chinese adolescents. Sleep. 2008;31(2):185-94.

22. Loessl B, Valerius G, Kopasz M, Hornyak M, Riemann D, Voderholzer U. Are adolescents chronically sleep-deprived? An investigation of sleep habits of adolescents in the Southwest of Germany. Child Care Health Develop. 2008;34(5):549-56.

23. Liu XC, Zhou HB. Sleep duration, insomnia and behavioral problems among Chinese adolescents. Psychiatry Res. 2002;111(1):75-85.

24. Carskadon MA, Harvey K, Duke P, Anders TF, Litt IF, Dement WC. Pubertal changes in daytime sleepiness. Sleep. 2002;25(6):453-60.

25. Siomos KE, Avagianou PA, Floros GD, Skenteris N, Mouzas OD, Theodorou K, et al. Psychosocial correlates of insomnia in an adolescent population. Child Psychiatry Hum Develop. 2010:41(3):262-73.

26. Jarrin DC, McGrath JJ, Quon EC. Objective and subjective socioeconomic gradients exist for sleep in children and adolescents. Health Psychol. 2014;33(3):301-5. 